\title{
PacBio single molecule long-read sequencing provides insight into the complexity and diversity of the Pinctada fucata martensii transcriptome
}

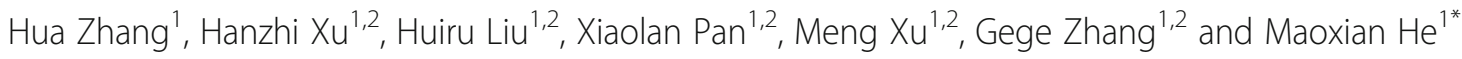

\begin{abstract}
Background: The pearl oyster Pinctada fucata martensii is an economically valuable shellfish for seawater pearl production, and production of pearls depends on its growth. To date, the molecular mechanisms of the growth of this species remain poorly understood. The transcriptome sequencing has been considered to understanding of the complexity of mechanisms of the growth of $P$. f. martensii. The recently released genome sequences of $P$. $f$. martensii, as well as emerging Pacific Bioscience (PacBio) single-molecular sequencing technologies, provide an opportunity to thoroughly investigate these molecular mechanisms.

Results: Herein, the full-length transcriptome was analysed by combining PacBio single-molecule long-read sequencing (PacBio sequencing) and Illumina sequencing. A total of $20.65 \mathrm{~Gb}$ of clean data were generated, including 574,561 circular consensus reads, among which 443,944 full-length non-chimeric (FLNC) sequences were identified. Through transcript clustering analysis of FLNC reads, 32,755 consensus isoforms were identified, including 32,095 high-quality consensus sequences. After removing redundant reads, 16,388 transcripts were obtained, and 641 fusion transcripts were derived by performing fusion transcript prediction of consensus sequences. Alternative splicing analysis of the 16,388 transcripts was performed after accounting for redundancy, and 9097 gene loci were detected, including 1607 new gene loci and 14,946 newly discovered transcripts. The original boundary of 11,235 genes on the chromosomes was corrected, 12,025 complete open reading frame sequences and 635 long non-coding RNAs (LncRNAs) were predicted, and functional annotation of 13, 482 new transcripts was achieved. Two thousand three hundred eighteen alternative splicing events were detected. A total of 228 differentially expressed transcripts (DETs) were identified between the largest (L) and smallest (S) pearl oysters. Compared with the $S$, the $L$ showed 99 and 129 significantly up-and down-regulated DETs, respectively. Six of these DETs were further confirmed by quantitative real-time RT-PCR (RT-qPCR) in independent experiment.
\end{abstract}

Conclusions: Our results significantly improve existing gene models and genome annotations, optimise the genome structure, and in-depth understanding of the complexity and diversity of the differential growth patterns of P. f. martensii.

Keywords: Pinctada fucata martensii, PacBio sequencing, Alternative splicing, LncRNAs, Differentially expressed transcripts

\footnotetext{
* Correspondence: hmx2@scsio.ac.cn

'CAS Key Laboratory of Tropical Marine Bio-resources and Ecology, Guangdong Provincial Key Laboratory of Applied Marine Biology, South

China Sea Institute of Oceanology, Chinese Academy of Sciences, Guangzhou 510301, China

Full list of author information is available at the end of the article
}

C C The Author(s). 2020 Open Access This article is licensed under a Creative Commons Attribution 4.0 International License, which permits use, sharing, adaptation, distribution and reproduction in any medium or format, as long as you give appropriate credit to the original author(s) and the source, provide a link to the Creative Commons licence, and indicate if changes were made. The images or other third party material in this article are included in the article's Creative Commons licence, unless indicated otherwise in a credit line to the material. If material is not included in the article's Creative Commons licence and your intended use is not permitted by statutory regulation or exceeds the permitted use, you will need to obtain permission directly from the copyright holder. To view a copy of this licence, visit http://creativecommons.org/licenses/by/4.0/. The Creative Commons Public Domain Dedication waiver (http://creativecommons.org/publicdomain/zero/1.0/) applies to the data made available in this article, unless otherwise stated in a credit line to the data. 


\section{Background}

Pincata fucata martensii is one of the most common oysters used for the production of seawater pearls, food and drugs. It is also one of the most useful animals for studying biominerals, hence it is often used as a model system to investigate the molecular basis of biomineralisation $[1,2]$. The growth, yield and quality of $P . f$. martensii is affected by various exogenous and endogenous factors, such as food availability [3], ocean acidification [4], temperature [5] and others. In recent years, increased mortality and slow growth have caused a distinct decline in pearl production due to a worsening aquaculture environment and aquatic diseases $[6,7]$. However, limited information exists on the molecular mechanisms that regulate the growth and development of this species. In recent years, molecular approaches such as linkage maps [8], transcriptomics, and proteomics [9] have been applied to reveal growth traits and guide the molecular breeding of various bivalves. Thus, a comprehensive understanding of the mechanisms of growth and development is required to improve pearl production.

RNA sequencing (RNA-seq) has become a powerful technique for investigating gene expression profiles and revealing signal transduction pathways in a wide range of biological systems [10]. In the past few years, substantial effort has been invested in genetic and genomic research related to $P$. f. martensii. In particular, RNA-seq has yielded new information at both the transcriptome $[11,12]$ and genome $[13,14]$ level. RNA-seq has shaped our understanding of many aspects of biology, such as revealing the extent of mRNA splicing and the regulation of gene expression. Although the genome sequence of $P . f$. martensii has been completed recently [14], the gene structure still needs to be optimized and perfected. Due to the limitation of short sequencing reads, it is difficult to accurately predict full-length (FL) splice isoforms [15]. Additionally, the extent of alternative splicing (AS) and transcriptome diversity remains largely unknown. Recently, the Pacific Bioscience (PacBio) Single Molecule Real Time Sequencing (SMRT) technique can overcome the limitation of short read sequences, enabling the detection of novel or rare splice variants that are crucial for post-transcriptional regulatory mechanisms, and increasing transcriptome diversity and functional complexity [16-18]. The PacBio single-molecule approach eliminates the need for sequence assembly, facilitates the accurate elucidation of FL transcripts and primary-precursor-mature RNA structures, and provides a better understanding of RNA processing due to its ability to sequence reads up to $50 \mathrm{~kb}[17,19]$. However, PacBio sequencing also has its own limitations, such as high sequencing error rates and low throughput [20, 21]. Fortunately, PacBio sequencing and Illumina sequencing are highly complementary to each other [22]. To address these issues, we herein propose a hybrid sequencing strategy that can provide more accurate information and generate more data in terms of volume of $P . f$. martensii than either technique alone.

In shellfish, understanding the differences between individuals is very important for developing strategies in breeding. Screening for growth-related candidate genes has helped advance molecular genetics and breeding [23, 24]. Growth of oysters were regulated by a series of genes associated with protein synthesis, signal transduction and metabolism $[9,11]$. Thus, identification of various differentially expressed genes involved in individual differences can provide insights into the growth mechanism, and develop suitable molecular markers for breeding [25]. Because growth mechanisms are complex and relate to many physiological processes, growth-related molecules derived from oysters have been studied using Illumina sequencing $[11,24,26]$. However, PacBio sequencing can provide further information on transcript diversity, including alternative splicing and alternative polyadenylation $[15,20]$. Combined with PacBio sequencing and Illumina sequencing, more gene isoforms could be detected, revealing functional variety $[18,27]$.

In order to better explore the growth differences between largest and smallest pearl oyster groups, we performed PacBio sequencing and Illumina sequencing. The results may permit reannotation of the transcriptome, improve whole-genome annotation, optimise the genome structure, and provide a valuable genetic resource for further studies of pearl oysters growth.

\section{Results}

\section{PacBio single molecule long-read sequencing data} analysis

Full-length cDNA sequences are important for correct annotation and identification of authentic transcripts from animal tissues. To generate a high quality transcriptome for $P . f$. martensii, we constructed $1-6 \mathrm{~kb}$ libraries and performed PacBio SMRT sequencing, which provides single-molecule, full-length transcript sequencing. A total of $2.65 \mathrm{~Gb}$ of clean reads were obtained. The Circular Consensus (CCS) library included 1,589, 889,145 bp with a mean length of 2767 bp (Table 1). A total of 574,561 CCS reads were obtained after filtering with SMRTLink (4.0). In total, 54,400 high-quality isoforms were identified, with 443,944 full-length reads (77.27\% of total CCS reads). In addition, 32,755 consensus isoforms were obtained, including 655 low-quality and 32,095 high-quality isoforms. The average consensus isoform read length was $2708 \mathrm{bp}$, and the density distribution of full length reads non-chimeric (FLNC) read length is shown in Fig. 1. Meanwhile, Illumina sequencing library was used to correct errors for further improve the accuracy of consensus reads. Using Illumina 
Table 1 The PacBio SMRT sequencing information of P. f. martensii

\begin{tabular}{ll}
\hline Category & Dataset \\
\hline Read bases of Circular Consensus (CCS) & $1,589,889,145$ \\
Number of ccs & 574,561 \\
Number of undesired primer reads & 80,026 \\
Number of undesired poly-A reads & 363,918 \\
Number of filtered short reads & 398 \\
Number of full-length non-chimeric reads & 443,944 \\
Full-length non-chimeric percentage (FLNC\%) & $77.27 \%$ \\
Number of consensus isoforms & 32,755 \\
Average consensus isoforms read length & 2708 \\
Number of polished high-quality isoforms & 32,095 \\
Number of polished low-quality isoforms & 655 \\
Gene loci & 9097 \\
New gene loci & 1607
\end{tabular}

sequencing, 152 million paired-end reads were sequenced. We used Proovread [28] to correct the FLNC reads based on the Illumina sequencing. A total of 16, 388 non-redundant transcripts were generated. BUSCO v3.0 (Benchmarking Universal Single Copy Orthologs) was utilized to determine completeness of our transcript dataset. The results showed that 41.3\% (125 genes) were complete single-copy BUSCOs, 21.5\% (65 genes) were complete duplicated BUSCOs, 6.6\% (20 genes) were fragmented BUSCO archetypes, and 30.6\% (93 genes) were missing BUSCOs entirely.

\section{Improving $P$. f. martensii genome annotation by PacBio sequencing}

Due to the limitations of the short read sequencing, annotation of the selected reference genome may not be sufficiently accurate, hence it is necessary to optimise the genetic structure of the original annotation. The PacBio technique has the advantage of sequencing length, and has been employed toward the optimisation of gene structure and the discovery of new transcript isoforms. The positions of 11,235 genes in the genome was optimised by the PacBio technique (Additional file 1: Table S1a, b), and 9097 gene loci were detected, of which 1607 were new gene loci. Gene fusion is caused by somatic chromosomal rearrangement, and fusion transcripts are related to the splicing machinery [29]. Herein, 641 fusion genes were identified in the PacBio library, and were validated using transcriptome datasets. The majority of these transcripts were mapped to the first and ninth chromosomes, but the location of $44 \mathrm{fu}-$ sion genes was unknown (Additional file 2: Table S2a, b). The number of intra-chromosomal fusion transcripts was much lower than that of inter-chromosomal fusion genes in the circos map (Fig. 2). Coding region sequences and their corresponding amino acid sequences were analysed using TransDecoder software (v3.0.0) based on new transcripts obtained from AS. Comparison with the $P . f$. martensii genome identified 14,313 open reading frame (ORFs), of which 12,025 complete ORFs were generated by PacBio sequencing. Meanwhile, length distribution of the encoded protein sequence for each complete ORF region was mapped, and the results are shown in Fig. 3a. Transcription factors (TFs) are essential for regulation of gene expression. Based on the animalTFDB 2.0 database, 836 transcripts were predicted to be TFs. The main TFs identified in this work belong to the ZBTB, zf-C2H2, Miscellaneous, Homeobox and bHLH families (Fig. 3b).

\section{Putative molecular marker detection}

Transcripts longer than $500 \mathrm{bp}$ were screened to analyse SSR transcripts using the MIcroSAtellite identification tool (MISA). The total size of examined sequences was $44,854,919 \mathrm{bp}$, the total number of identified SSRs was 8061, and the number of SSR-containing sequences was 5303 from 16,127 FL transcripts. Perfect SSRs included 6366 mono-nucleotide SSRs, 936 di-nucleotide SSRs, 634 tri-nucleotide SSRs, 109 tetra-nucleotide SSRs, 15 penta-nucleotide SSRs and one hexa-nucleotide SSR. The number of SSRs gradually decreased with an increasing number of repeated SSR motifs. Mononucleotides showed the highest density. All SSRs are listed in Additional file 3: Table S3.

\section{Alternative polyadenylation (APA) and alternative splicing (AS) analysis}

Polyadenylation is an important co-transcriptional modification in most eukaryotic transcripts. Alternative polyadenylation regulates gene expression and enhances the complexity of the transcriptome. A total of 7216 genes detected by the APIS pipeline have at least one poly (A) site, and 2142 genes have at least two or more poly (A) sites (Fig. 4a; Additional file 4: Table S4). Mature mRNAs are generated by a variety of splicing methods, and are translated into different proteins to increase biological complexity and diversity. The most important advantages of PacBio sequencing is its ability to identify AS events. A total of 2318 AS transcripts were predicted from the PacBio sequence data using AStalavista analysis, of which 177 AS transcripts were not annotated in the published version of the $P . f$. martensii genome (Additional file 5: Table S5a, b). Five kinds of AS events were identified (Fig. 4b); mutually exclusive exons (11.04\%), intron retention (25.19\%), exon skipping (37.75\%), alternative $5^{\prime}$ splice sites (14.67\%) and alternative 3 ' splice sites (11.35\%). Exon skipping and intron retention events were much more abundant than the 


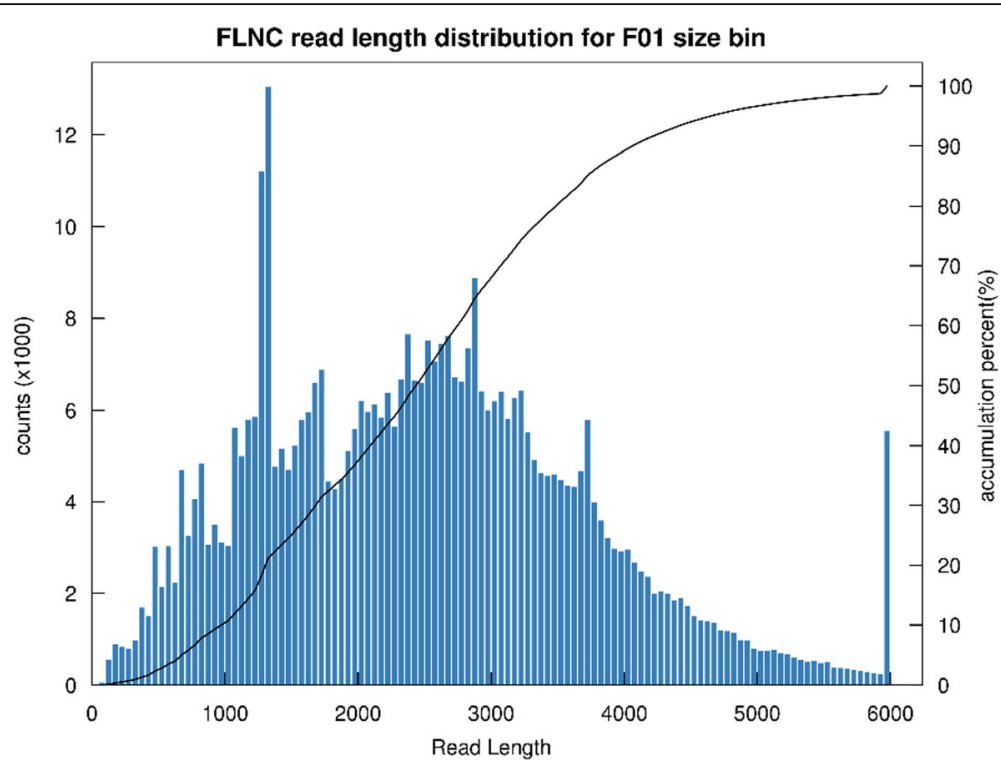

Fig. 1 Density distribution of full length readsnon-chimeric (FLNC) read length obtained by SMART sequencing

other three types. The location of AS transcripts in the genome was described for all but 177 AS transcripts.

\section{Functional annotation of transcripts}

The newly identified transcripts sequence were scanned against the NCBI non-redundant protein sequences (NR), Protein family (Pfam), Clusters of Orthologous Groups of proteins (KOG/COG/eggNOG), a manually annotated and reviewed protein sequence database (Swiss-Prot), Kyoto Encyclopedia of Genes and Genomes (KEGG) and Gene Ontology (GO) databases using BLAST 2.2.26 software to obtain annotation information for each transcript. The number of transcripts annotated in each database is shown in Fig. 5a. In total, 4386 transcripts were annotated in the COG database, 5160 were annotated in GO, 7067 were annotated in KEGG, 9337 were annotated in KOG, 11,371 were annotated in Pfam, 8204 were annotated in Swiss-Prot, 11,879 were annotated in eggNOG, and 13,309 were annotated in NR. Moreover, 13,482 transcripts were annotated in all databases. Meanwhile, new transcripts obtained from AS analysis were functionally annotated. Based on NR annotation, species homologous with $P$. f. martensii were predicted by sequence alignment. Crassostrea gigas and Crassostrea virginica were the closest matching genomes, followed by Mizuhopecten yessoensis (Fig. 5b). In GO annotation (Fig. 5c), transcripts were classified into three main GO categories; cellular component (CC), molecular function (MF) and biological process (BP). In the three main categories, metabolic process (BP) (4663), catalytic activity (MF) (4198) and cell part (CC) (2308) were the most enriched subcategories, respectively. Besides, the published version of P.f.martensii genome annotations contains 32,937 protein-coding gene models [14]. In the transcriptome database, 1028 gene are not annotated in the genome. To assess the presence of these unannotated genes, we conducted BLAST analyses, 516 were found in the blastx search against Swiss-Prot proteins, 986 in NR, 245 in COG database,309 in GO, 416 in KEGG, 578 in KOG,804 in eggNOG and 781 in Pfam (Additional file 6: Table S6).

\section{LncRNA prediction}

LncRNAs play an important role in regulating gene expression in most eukaryotes. Based on Coding Potential Calculator (CPC), Coding-Non-Coding Index (CNCI), Pfam protein structure domain and Coding Potential Assessment Tool (CPAT) analyses, the number of lncRNAs transcripts was 4194, 839, 3512, and 1713, respectively (Additional file 7: Table S7a, b), across all chromosomes. Additionally, 635 lncRNAs transcripts were identified in all analyses (Fig. 6a). Identification of lncRNAs was classified based on their position in the reference genome and annotation information. The 635 lncRNAs included 120 sense-lncRNAs, 21 intronic-lncRNAs, 17 antisenselncRNAs and 446 lncRNAs (Fig. 6b). To investigate the functions of lncRNAs, we identified the potential targets of IncRNAs based on positional relationships between lncRNAs and mRNAs, and correlation analysis between lncRNAs and mRNA expression in samples (Additional file 8: Table S8). Mapping lncRNAs to chromosomes revealed that they have a distribution similar to that of mRNAs (Fig. 2).

\section{Differentially alternative splicing (AS) and differentially expressed transcripts (DETs) analysis}

A single gene can generate functionally distinct mRNAs and diverse protein isoforms by recognition of exons 


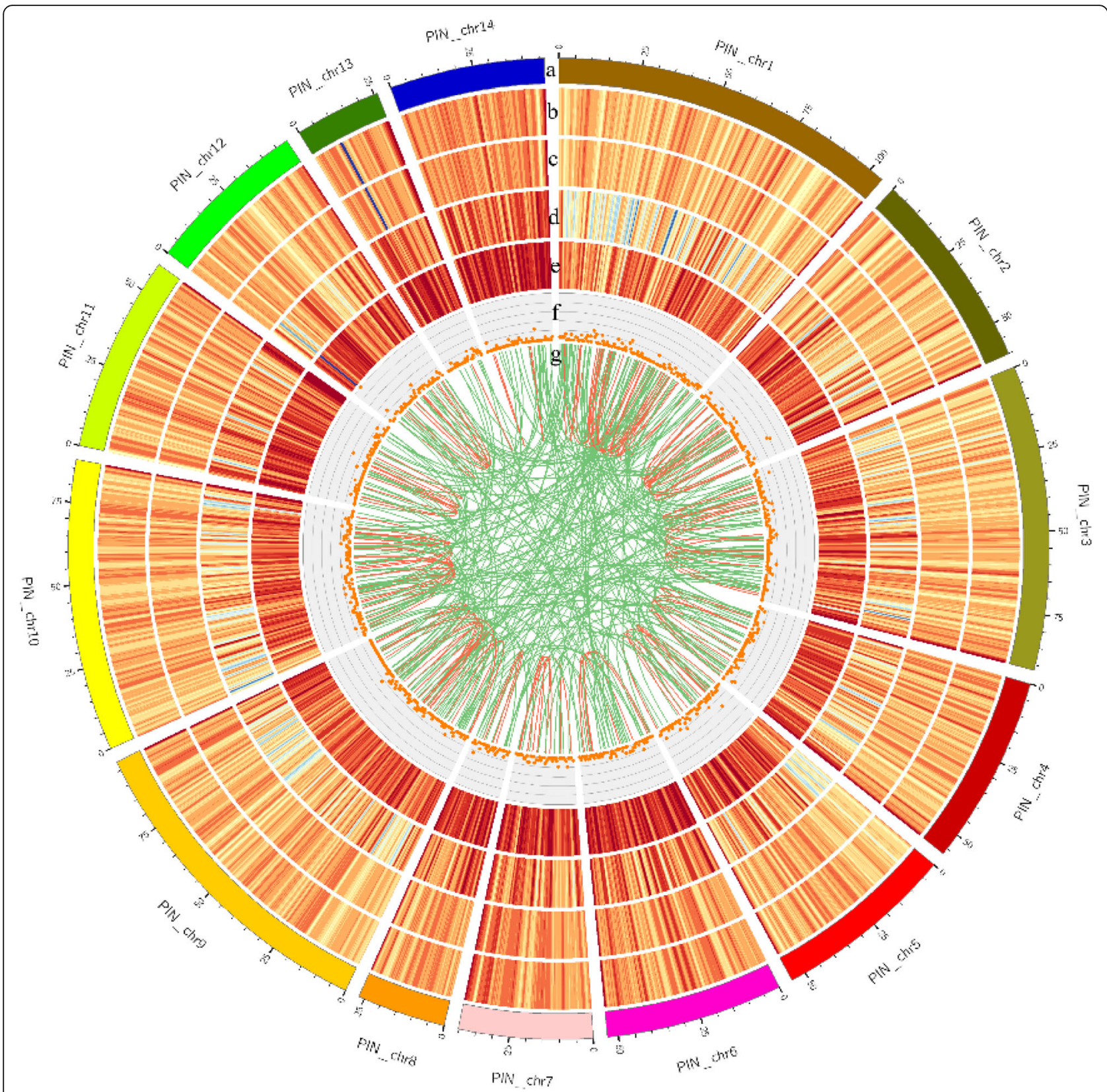

Fig. 2 CIRCOS visualisation of the distribution of different data at the genome-wide level. a: Pincata fucata martensii chromosomes. b: Gene density of the reference genome. c: Density of genes predicted from the PacBio data. $\mathbf{d}$ : Transcript density in the genome. $\mathbf{f}$ : Long non-coding RNA (IncRNA) distribution in chromosomes. g: Fusion transcript distribution. Intra-chromosome data are coloured red inter-chromosome (green)

and splice sites during splicing. We performed differentially variable splicing analysis between the $\mathrm{L}$ (L01, L02, L03 represent three subgroups from L groups) and S (S01, S02, S03 represent three subgroups from S groups) groups using RNA-seq. The expression correlation for S01 sample oysters was inconsistent with that of S02 and S03. Hence, data from the S01 sample were removed. Interestingly, the data showed that the number of the five basic types of AS models (except for A3SS in L groups) was much higher than for S groups; 144 significantly differential AS events in S groups were detected using junction counts alone, including 83 in SE, 44 in MEX, four in A5SS, three in A3SS and ten in RI. A total of 147 significantly differential AS events in L groups were identified using both junction counts and reads on targets, including 87 in SE, 42 in MEX, four in A5SS, five in A3SS and nine in RI. The number of AS events in $\mathrm{L}$ and $\mathrm{S}$ groups are shown in Additional file 9: Table S9. 


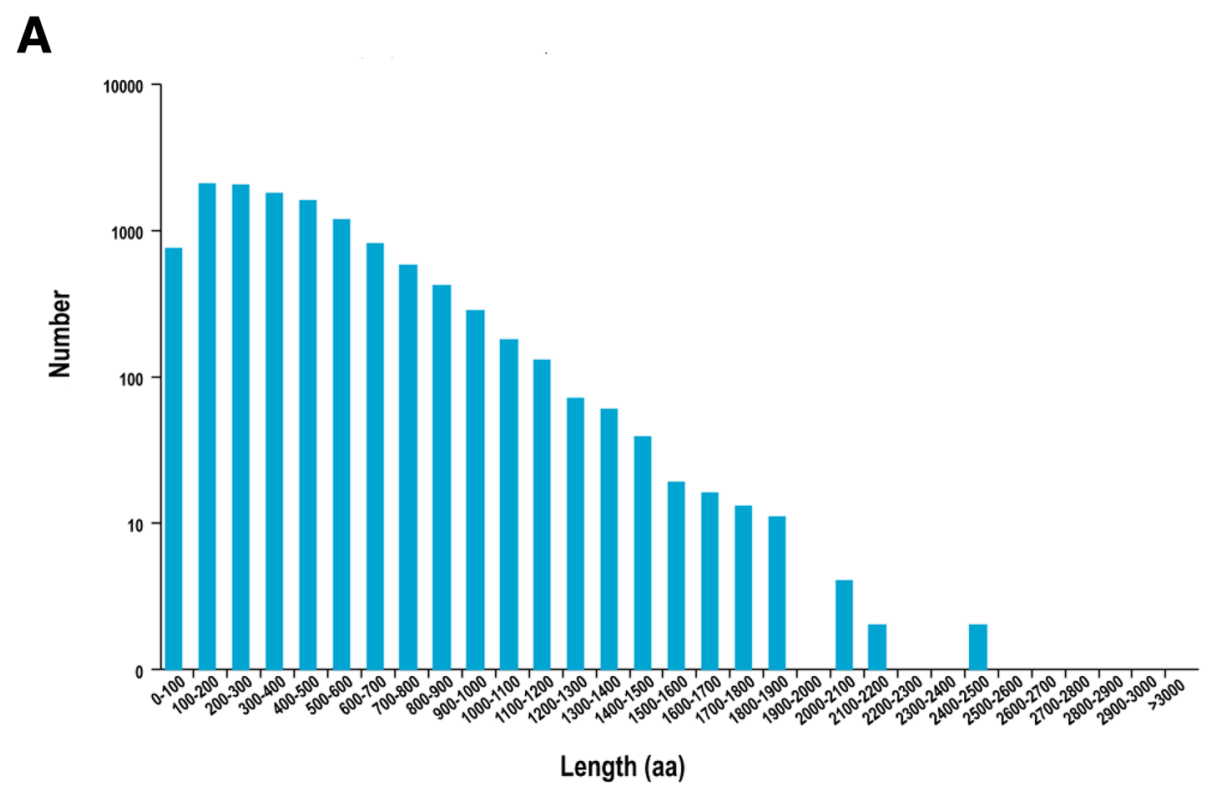

\section{B}

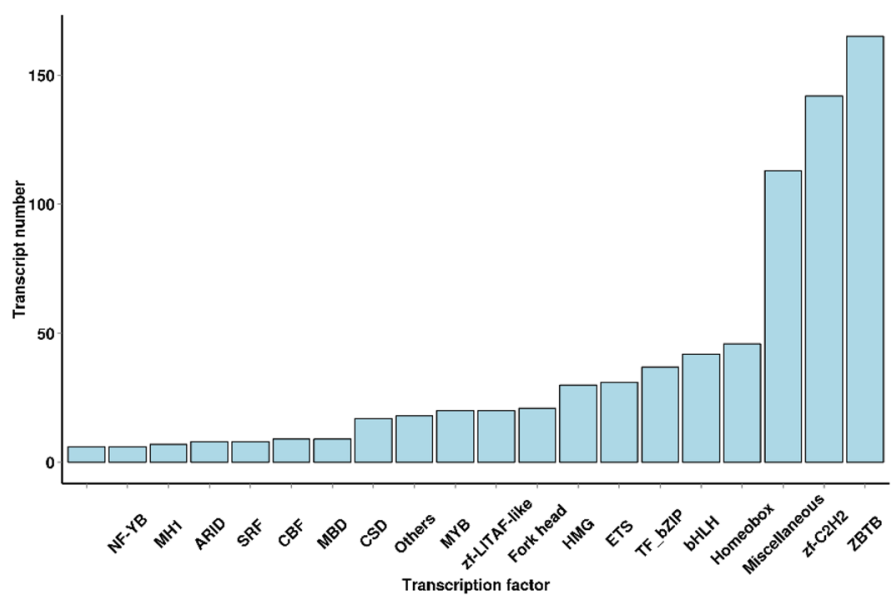

Fig. 3 Length distribution of complete open reading frames (cds) (a) and type distribution of transcription factors (b)

Transcript expression displays temporal and spatial specificity. Post-transcriptional processing of precursor mRNAs leads to transcript diversity, and hence diverse biological functions. We performed Illumina sequencing to search for transcripts shared between $\mathrm{L}$ and $\mathrm{S}$ groups. The FPKM method was used to estimate DETs. Our analysis yielded 228 DETs $(|\log 2 \mathrm{FC}| \geq 2$, FDR $<0.01)$, among which 99 were upregulated and 129 were down-regulated in the pairwise groups (Additional file 10: Table S10). Differences in expression levels of transcripts in the pairwise comparisons are shown in a volcano plot (Fig. 7a). Interestingly, KEGG pathway analysis showed that DETs were mainly assigned to metabolism, followed by genetic information processing, cellular processes, environmental information processing, human diseases, and organismal systems (Fig. 7b).

Six transcripts were selected for validation by RTqPCR. These transcripts were PB.2597.2 (proliferationassociated protein 2G4), PB.3595.2 (neural cell adhesion molecule 1), PB.1291.5 (monocarboxylate transporter 9), PB.1690.1 (cell division cycle 16-like protein), PB.2529.1 (fatty acid-binding protein) and Pma_10001161 (mineralisation-related protein 1). The RT-qPCR results showed that four transcripts (PB.2597.2, PB.3595.2, PB.1291.5 and PB.1690.1) were significantly up-regulated in $\mathrm{S}$ groups. However, the RT-qPCR and RNA-seq results for PB.2529.1 and Pma_10001161 were inconsistent. They did not show a significant difference by RTqPCR (Fig. 8). 
A

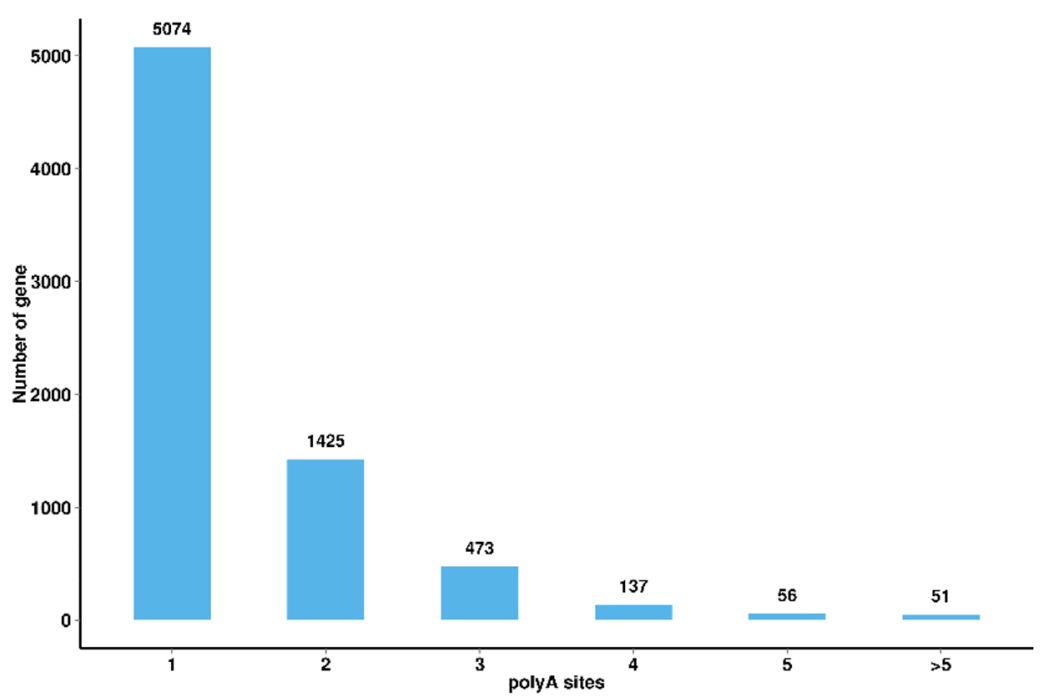

B

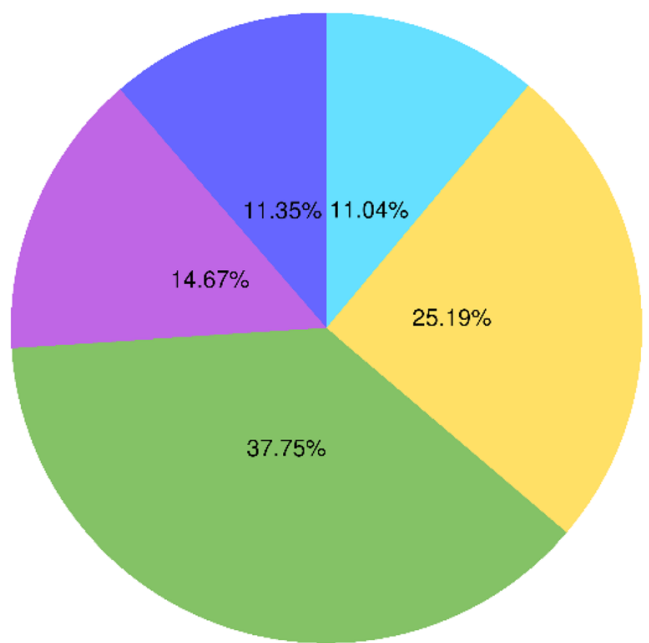

Alternative 3 ' splice site

Alternative 5' splice site

Exon skipping

Intron retention

Mutually exclusive exon

Fig. 4 Characterisation of poly (A) sites and alternative splicing (AS) events. a: Distribution of the number of poly (A) sites per gene. b: Number of alternative splicing (AS) events

\section{Discussion}

\section{PacBio sequencing can optimize genome structure}

Due to the limitations of short read sequencing, annotation of the reference genome is often not sufficiently accurate. In our present work, a hybrid sequencing approach was used to optimise the genetic structure of the original annotation. The original boundary of 11,235 genes on the chromosomes was corrected. Additionally, 1607 gene loci were newly discovered in the P. f. martensii genome, and 14,946 transcripts were newly identified that were absent from the known transcriptome annotation. Thus, PacBio sequencing can be an effective strategy for improving the accuracy and quality $P$. f. martensii genome annotation information.

PacBio sequencing reveals complexity and diversity in the P. f. martensii transcriptome

In eukaryotes, transcripts are highly complex and diverse since precursor mRNAs are subjected to multiple posttranscriptional modification processes, such as AS and 
A

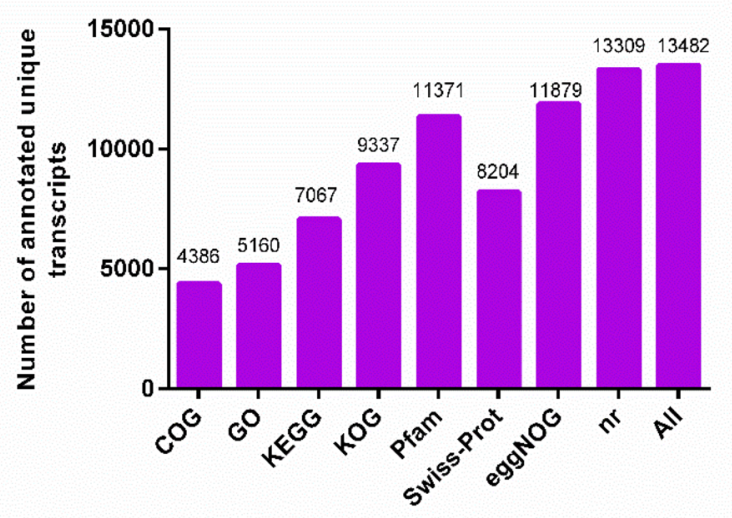

Annotation againsted different databases

B

NR Species distribution

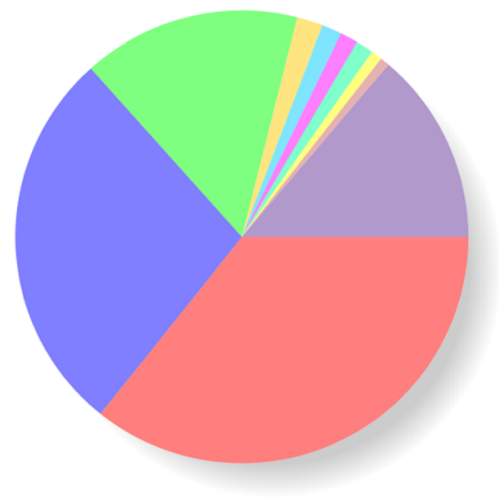

Crassostrea gigas [9790 35.74\%]

Crassostrea virginica [7571 27.64\%]

Mizuhopecten yessoensis [4259 15.55\%]

Lottia gigantea [508 1.85\%]

Pinctada fucata martensii [391 1.43\%]

Lingula anatina [350 1.28\%]

Lasius niger [342 1.25\%]

Lucilia cuprina [220 0.80\%]

Exaiptasia pallida [186 0.68\%]

Other [3775 13.78\%]

C

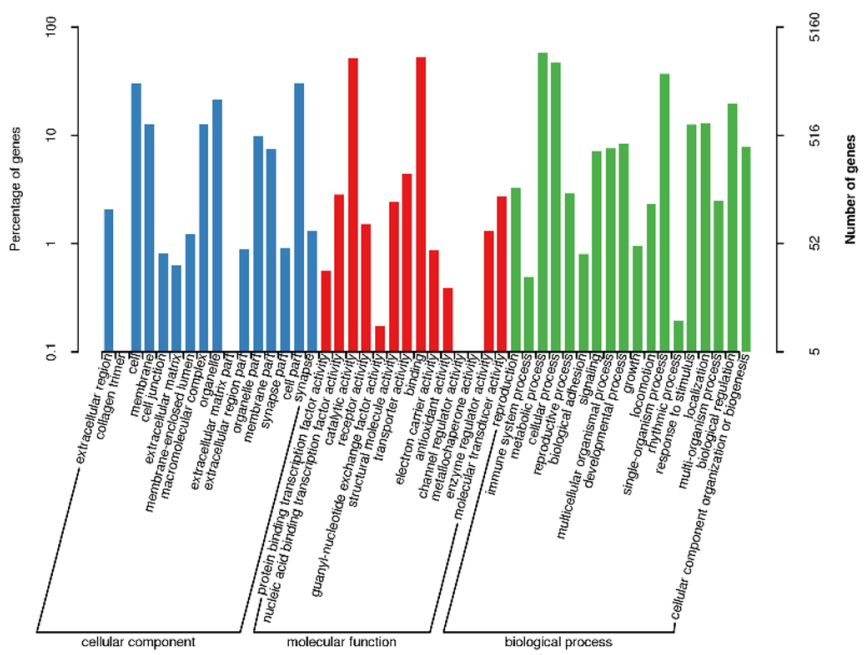

Fig. 5 Functional annotation of non-redundant transcripts in public databases. a: Number of full-length transcripts annotated in COG, GO, KEGG $\mathrm{Nt}$, Nr, SwissProt, KOG, and Pfam databases. b: GO classification of the putative functions of unique transcripts. c: Distribution of homologous species annotated in the NR database 


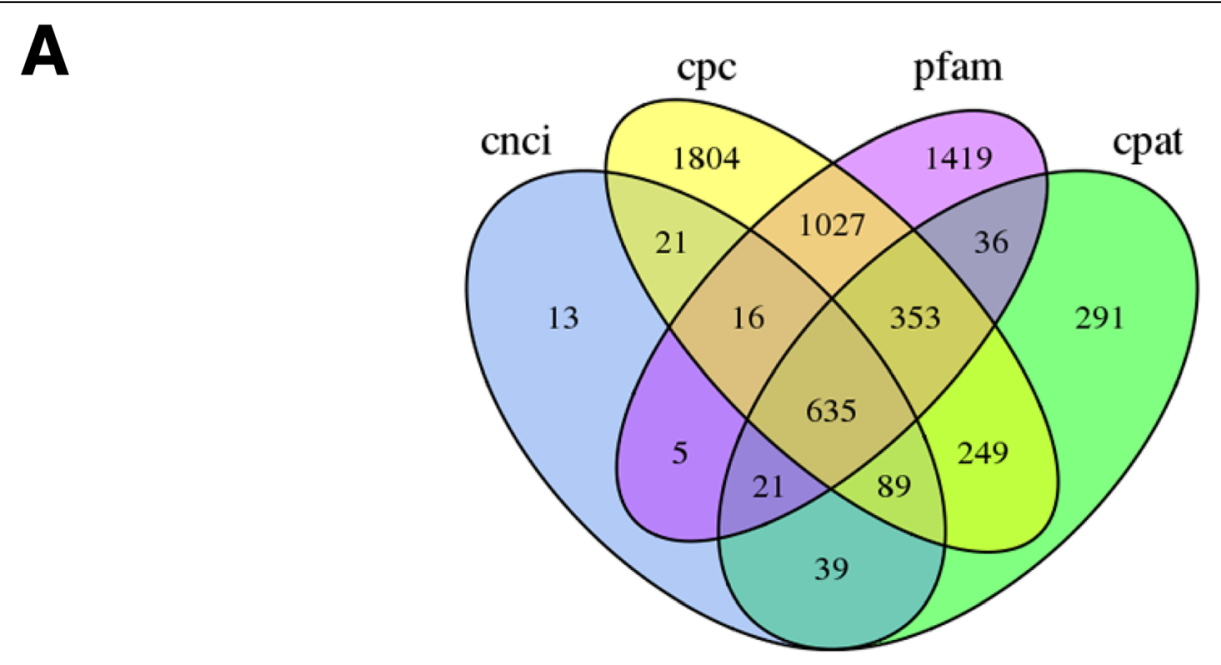

B

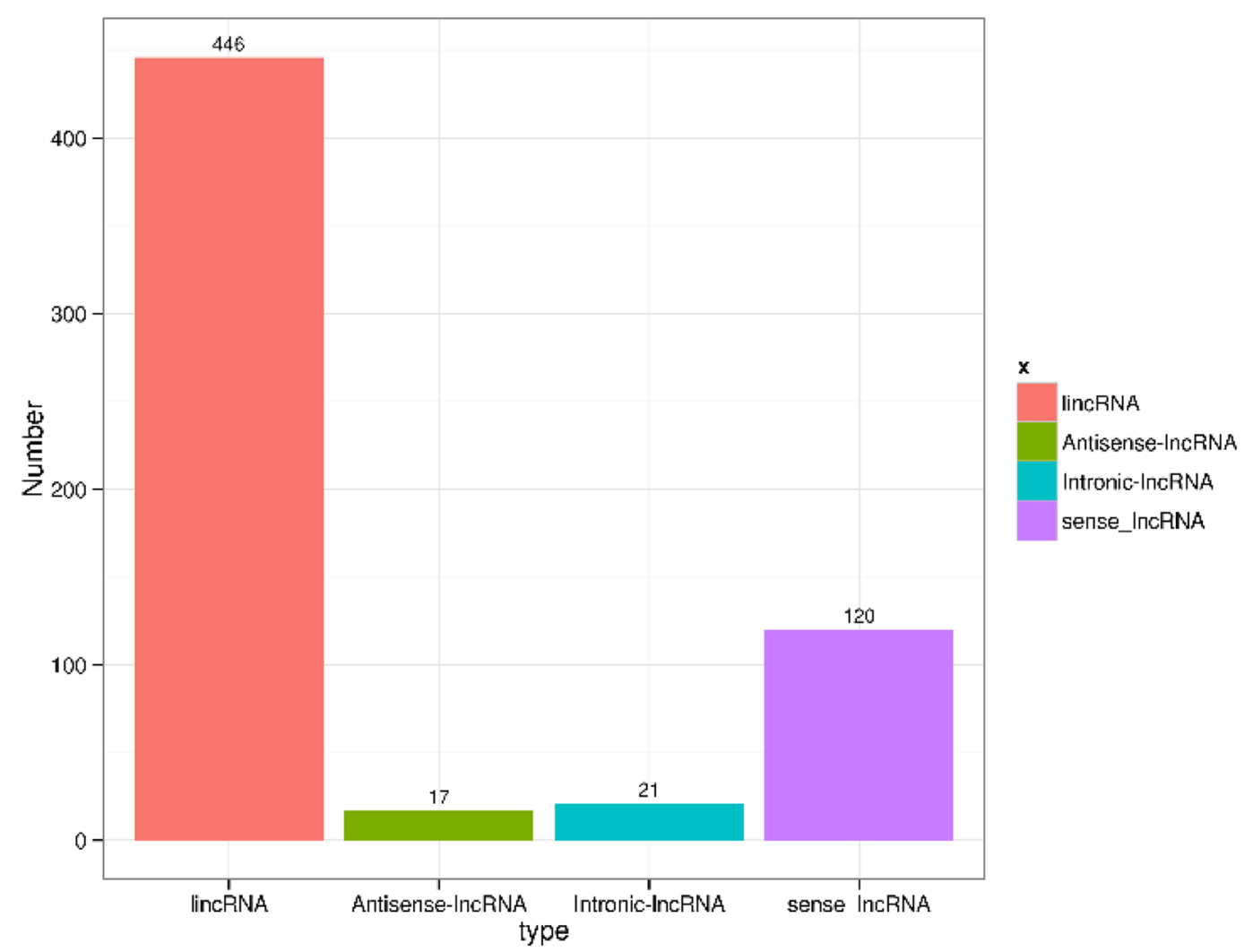

Fig. 6 Prediction of IncRNAs in Pincata fucata martensii. a: Candidate IncRNAs identified using CNCI, CPC, Pfam and CPAT. Non-overlapping areas indicate the number of IncRNAs identified by the single tool; overlapping areas indicate the total number of IncRNAs identified by multiple tools. b: Types of IncRNAs 


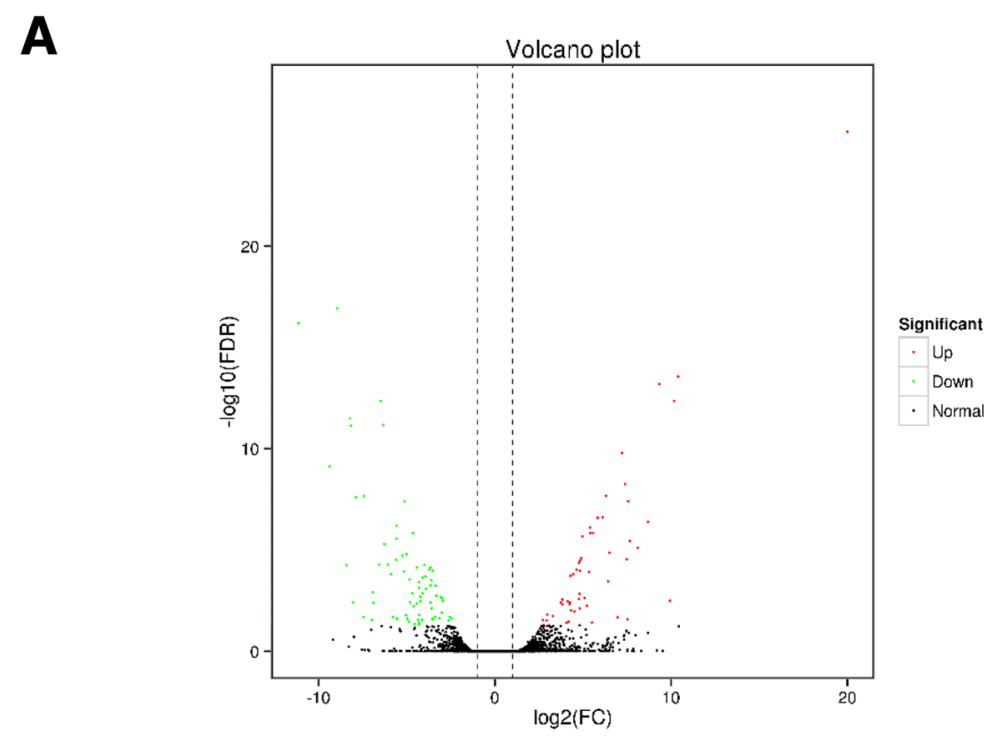

B

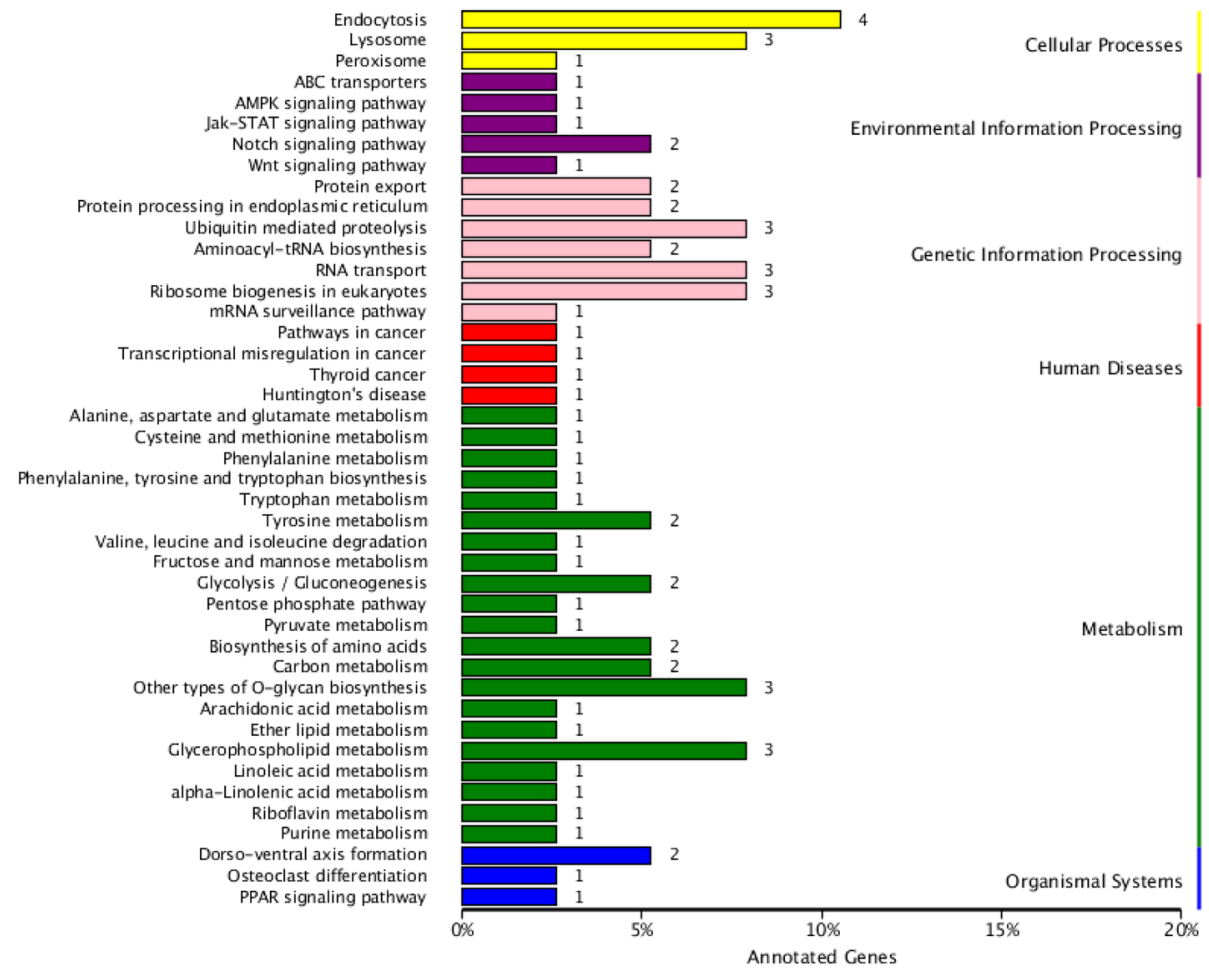

Fig. 7 Analysis of DETs between $S$ and L subgroups. a: volcano plot of transcripts. Each point in the volcano plot represents a transcript, the abscissa represents the logarithm of the difference in expression of a transcript in the two samples, and the ordinate represents the negative logarithm of the statistical significance of the change in the expression of the transcript. Green dots represent down-regulated DETs, red dots represent up-regulated DETs, and black dots represent non-DETs. b: KEGG classification map of DETs. The ordinate represents the KEGG metabolic pathway, and the abscissa represents the number of transcripts annotated to the pathway and the percentage of transcripts in the annotation 
PB.3595.2

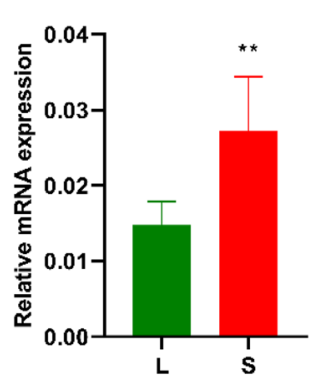

PB.1291.5
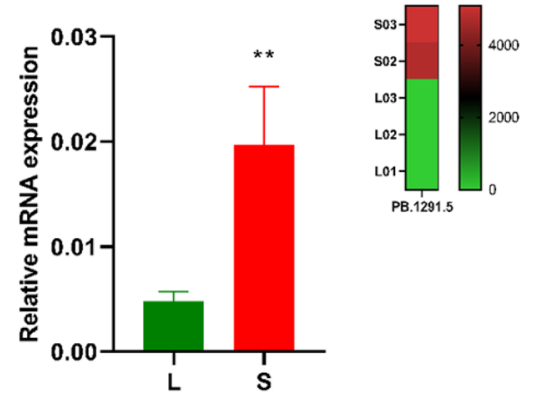

PB.2597.2
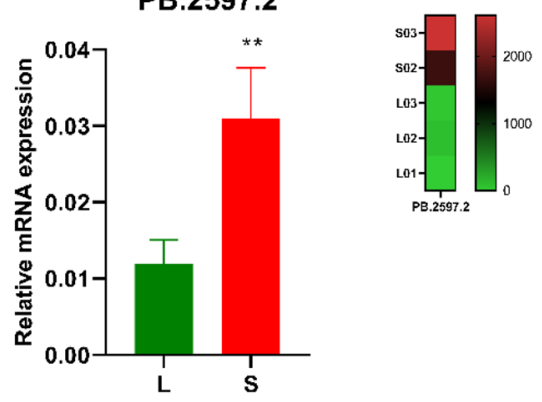

Pma_10001161

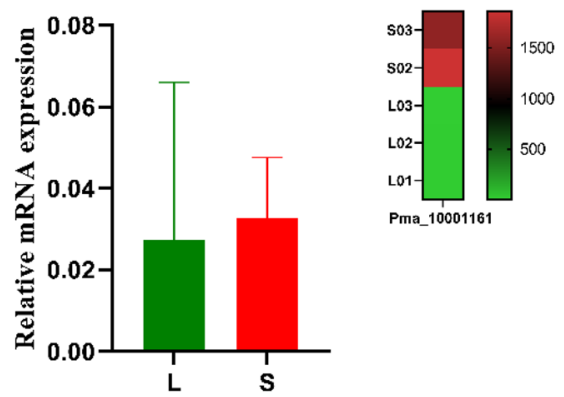

Fig. 8 Relative mRNA expression profiles of six transcripts in Pincata fucata martensii selected for verification by quantitative real-time RT-PCR analysis. The number of transcripts in the transcriptome is shown in the heat map. Statistical changes were determined by Student's $t$ tests (two-tailed) and are denoted as ${ }^{* *} p<0.002$ and ${ }^{* *} p<0.0002$

APA [30, 31]. RNA-seq has showed that eukaryotic transcriptomes are highly complex due to posttranscriptional processing of precursor mRNAs by AS and APA [15, 32]. Herein, we identified AS and APA events from $P . f$. martensii. Differential polyadenylation of mRNAs generates transcript isoforms, and APA is necessary for RNA transport, localisation, stability and translation [30, 33]. Illumina sequencing has been widely used for gene expression analysis. However, it is unable to accurately detect APA, AS, fusion genes and gene families [34, 35]. The unigenes obtained in this study displayed high functional annotation rates of up to $82.26 \%$. This may be attributed to the long-read sequences obtained using the PacBio single-molecule approach, which offers a considerable advantage for transcriptome-wide identification of full-length splice isoforms and other forms of post-transcriptional regulatory events such as APA [15]. PacBio sequencing technology can capture FL transcripts without the need for further assembly, making it more popular for 
transcriptome analysis [36, 37]. FL transcripts can also provide deep insight into transcriptome characterisation, and can greatly improve the accuracy of genome annotation [29]. However, PacBio sequencing has a higher error rate and must be corrected using Illumina sequencing reads [38] and/or CCS reads [39, 40]. By combining PacBio sequencing and Illumina sequencing, FL transcriptome data has great potential for the discovery of novel or previously unrecognised protein-coding genes and isoforms [41]. In the present work, 12,025 complete ORF sequences were obtained.

\section{Hybrid sequencing analysis reveals insight into the growth and development of $P$. f. martensii}

In the present work, 228 DETs were identified between $\mathrm{L}$ and $\mathrm{S}$ groups, of which 99 DETs were up-regulated and 129 down-regulated in L groups. We analysed the mRNA expression profiles of six growth and development-related genes, and four of the six genes were up-regulated in S groups. Monocarboxylate transporter (MCT) is an important transmembrane transporter involved in many physiological functions, such as the regulation of nutrient absorption, intracellular $\mathrm{pH}$, drug delivery and metabolism homeostasis [42-44]. Neuronal cell adhesion molecule 1 (NCAM 1) is a member of the immunoglobulin (Ig) superfamily of adhesion molecules, which are involved in binding between cells, helping cells stick to each other and their surroundings. NCAMs regulate bodyweight and energy homeostasis $[45,46]$. Proliferation-associated $2 \mathrm{G} 4$ proteins are a conserved family in eukaryotes that are involved in cell proliferation and differentiation $[47,48]$. Cell division cycle 16-like protein is one of the components of the anaphase-promoting complex involved in cell division [49]. Fatty acid-binding protein is an intracellular fatty acid carrier protein that plays an important role in the utilisation of fatty acids in cells [50]. Mineralizationrelated protein 1 is related to biomineralisation. Two of these transcripts were inconsistent between RT-qPCR and RNA-seq data. RNA-seq is a method of large-scale screening that reflects trends in gene expression in whole samples, but it does not guarantee that trends for each gene are consistent between RNA-seq and qPCR methods. Previous research reported that the majority of differentially expressed metabolites are up-regulated in small individuals [24, 51]. Additionally, some genes related to growth and development, shell formation, and reproduction are expressed more highly in $\mathrm{S}$ groups compared with L groups in pearl oyster [26]. This could be explained by the 'catch-up growth' phenomenon. Furthermore, immunisation may hinder growth [52]. Nutrients and energy ordinarily used for growth may be diverted to the generation of immune responses. Analysis of size in Pinctada maxima through transcriptomics indicated that low growth in small individuals could be partially explained by energy allocated for immune responses caused by stressors under certain environmental conditions [24]. In the present work, in addition to growth and development-related genes, some genes related to immune processes (PB.1364.11, PB.4993.4 and PB.3677.1) were differentially expressed between $\mathrm{L}$ and $\mathrm{S}$ groups. These results are in accordance with previous research [24]. Additionally, in our transcriptome databases, we identified numerous hypothetical proteins and uncharacterised protein displaying extremely significant differences. These proteins will be investigated in future research.

\section{Conclusion}

We present the full-length transcriptome of $P$. f. martensii using PacBio single-molecule long-read sequencing. The results improve existing genome annotations, optimise the genome structure, and in-depth revealing the complexity and diversity of the differential growth patterns of $P . f$. martensii individuals. The findings may prove valuable for dissection mechanisms of growth traits and guiding molecular breeding in P. f. martensii.

\section{Methods}

\section{Sample collection and RNA preparation}

Adult P. f. martensii were obtained from the Daya Bay Marine Biology Research Stations of the Chinese Academy of Sciences (Shenzhen, Guangdong, P.R. China). Seven tissues including mantle, digestive gland, gonad, adductor muscle, foot, heart and gill were harvested and stored in liquid nitrogen until RNA extraction. Total RNA from each tissue was extracted and purified using TRIzol reagent following the manufacturer's instructions (Magen, Guangzhou, China). RNA purity, concentration and integrity were assessed using an Agilent RNA 6000 nano kit and chips on a Bioanalyzer 2100 (Agilent Technologies, Santa Clara, CA, USA) and processed further for cDNA preparation. For PacBio sequencing, RNA samples from each tissue were mixed in an equal molarity for cDNA library construction. For Illumina sequencing, 100 one-year-old individuals were randomly selected from a Shenzhen population and shell length (SL) was measured, pearl oysters the top 9 and the bottom 9 in SL value were selected as largest individual (L group) and smallest individual (S group), respectively. The mantle tissue of each individual was collected and extracted for total RNA. RNAs from L group or S group were randomly divided into three subgroups, namely L01, L02, L03 or S01, S02, S03. Specifically, equal quantities of RNAs from three largest individual (L group) or smallest ( $\mathrm{S}$ group) individual were pooled into one mixed sample for cDNA library construction. 
Illumina sequencing library construction and sequencing Six double-stranded cDNA libraries were prepared using NEBNext ${ }^{\circ}$ Ultra $^{\text {Tu }}$ RNA Library Prep Kit according to the manufacturer's methods. Briefly, mRNA was enriched from total RNA using Oligo (dT)-attached magnetic beads and randomly interrupted by fragmentation and reverse transcribed into double-stranded cDNA. The purified double-stranded cDNA was subjected to terminal repair and then ligated to sequencing adapters. Fragments of $380 \sim 530 \mathrm{bp}$ were then selected, purified and subsequently PCR amplified to create the final cDNA library template for sequencing. The final library was quantitated using an Agilent 2100 Bioanalyzer instrument (Agilent). Six qualified libraries (L01, L02, L03; S01, S02, S03) were sequenced using an Illumina HiSeq 2000 System with pair-end 150 bp read length.

\section{PacBio sequencing library construction}

mRNA was isolated from $5 \mu \mathrm{g}$ of mixed total RNA using poly (T) oligo-attached magnetic beads. Fragmentation was treated with divalent cations under elevated temperatures in the NEBNext First Strand Synthesis Reaction Buffer $(5 \times)$. Full-length cDNA was synthesized by SMAR $\mathrm{Ter}^{\mathrm{m}}$ PCR cDNA Synthesis Kit (Clontech, CA, USA). Remaining overhangs were converted into blunt ends by exonuclease/polymerase activities. After adenylation of the 3' ends of the DNA fragments, hybridization was performed by ligating NEBNext adaptor with a hairpin loop structure. The size selection of the full-length cDNA and for building libraries of differently sized cDNA were performed by BluePippin ${ }^{\circ}$ (Sage Science, Beverly, MA, USA). The generated cDNA was then re-amplified using PCR, and the fragment size distribution was quantified using the Qubit fluorometer (Life Technologies, Carlsbad, CA, USA). The quality of the libraries was assessed using the Agilent Bioanalyzer 2100 system.

\section{PacBio sequencing processing}

PacBio sequencing was performed using a PacBio RS II instrument. Raw reads were processed into errorcorrected reads of inserts (ROIs) using the Iso-seq pipeline with full passes $\geq 0$ and predicted accuracy $>0.80$. Full-length transcripts were identified by searching for both $5^{\prime}$ and $3^{\prime}$ primer sequences and poly (A) tails in ROIs. Consensus isoforms and FL consensus sequences were obtained from Iterative Clustering for Error Correction using Quiver. High-quality FL transcripts were classified with post-correction accuracy $>9 \%$. FL consensus sequences were mapped to the reference genome using Genomic Mapping and Alignment Program (GMAP) [53], and mapped reads were further collapsed by the pbtranscript-ToFU package with min-coverage $=$ $85 \%$ and min-identity $=90 \%$. Differences in $5^{\prime}$ sequences were not considered when collapsing redundant transcripts and Illumina data were used for PacBio sequencing error correction. The integrity of de-duplicated transcriptomes was assessed using BUSCO v3.0 [54].

\section{Illumina sequencing data processing}

Raw data (raw reads) were firstly processed to remove adapters, reads containing poly- $\mathrm{N}$, and low-quality reads, generating clean data (clean reads). At the same time, Q20, Q30, GC-content and sequence duplication values were calculated for clean data. All downstream analysis was based on clean data of high quality. Clean reads were then mapped to the reference genome sequence. Only reads with a perfect match or one mismatch were further analysed and annotated based on the reference genome. Tophat 2.1.1 (http://ccb.jhu.edu/software/tophat/index. shtml) was used to map to the reference genome.

\section{Prediction of fusion transcripts}

The criteria for fusion candidates were (1) each transcript must be mapped to two or more distinct proteinencoding loci in the genome; (2) each mapped locus must align at least $5 \%$ of the transcript; (3) the combined alignment total coverage across all matched loci must be $\geq 99 \%$; (4) each mapped locus is at least $10 \mathrm{~kb}$ apart.

\section{Structural analysis of transcripts}

Transcripts were validated against known reference transcript annotations with the python library MatchAnnot (https://github.com/TomSkelly/MatchAnnot). Coding region sequences and their corresponding amino acid sequences were analysed using TransDecoder v3.0.0 (https://transdecoder.github.io/) software based on new transcripts obtained from alternative splicing. Transcription factors were identified using the animalTFDB 2.0 database [55]. Alternative polyadenylation (APA) analysis was conducted with TAPIS 1.2.1 (https://bitbucket.org/ comp_bio/tapis/overview), and AS events including IR, $\mathrm{ES}, \mathrm{AD}, \mathrm{AA}$ and MEE were identified by the AStalavista tool $3.0[56]$.

\section{Functional annotation and enrichment analysis}

The functions of non-redundant transcripts were annotated using the following databases: NR, Pfam, KOG/ COG/eggNOG, Swiss-Prot, KEGG and GO. Transcripts were subjected to enrichment analysis using GO implemented by the GOseq $R$ packages based on the Wallenius non-central hyper-geometric distribution, which can adjust for gene length bias. KEGG is a database resource for understanding biological systems and high-level functions. KOBAS 2.0 software [57] was used to identify KEGG pathways enriched with differentially expressed transcripts (DETs). GO and KEGG pathway enrichment analyses were assessed by Fisher's exact tests corrected with a false discovery rate (FDR) of $1 \%$. 


\section{Molecular marker identification}

Transcripts that were $>500 \mathrm{bp}$ in length were selected for SSR analysis with MIcroSAtellite identification tool (MISA v1.0) (http://pgrc.ipk-gatersleben.de/misa/misa. html). MISA can identify seven SSR types, namely mono-nucleotide, di-nucleotide, tri-nucleotide, tetranucleotide, penta-nucleotide, hexa-nucleotide, and compound SSR.

\section{Long-coding RNAs (IncRNAs) analysis}

Putative protein-coding RNAs were filtered using minimum length and exon number thresholds. Non-proteincoding RNA transcripts with more than two exons and longer than $200 \mathrm{bp}$ were sorted as lncRNA candidates using four computational approaches, namely Coding Potential Assessment Tool (CPAT), Coding-Non-Coding Index (CNCI), Coding Potential Calculator (CPC), and Pfam protein structure domain analysis (Pfam), all of which can distinguish protein-coding and non-coding transcripts. The identification of long-coding lncRNAs were chosen for predicting target genes based on location and co-expression relationship.

\section{Quantification of transcript expression levels and differential expression analysis}

Unigene expression levels were analysed based on short read datasets generated by the Illumina sequencing platform. Transcript levels were estimated using the fragments per kilobase of transcript per million (FPKM) method mapped using HISAT 2.0 (https://ccb.jhu.edu/ software/hisat2/index.shtml). Mantle tissue was used as the experimental tissue. Analysis of differential transcripts between two groups was performed using the DESeq R package (1.10.1) method [58]. DESeq provides statistical routines for determining differential expression in digital transcript expression data using a model based on the negative binomial distribution. The resulting $p$-values were adjusted using Benjamini and Hochberg's approach for controlling the FDR. Only genes with an absolute value of $|\log 2 \mathrm{FC}| \geq 2$ and FDR significance score $<0.01$ were used for subsequent differentially expressed analysis.

\section{Quantitative real-time PCR analysis of DETs}

DETs were selected to validate novel transcripts identified via RNA-seq. Five random individuals were sampled from largest or smallest groups, respectively. Total RNA was extracted from mantle tissue using TRIzol reagent and subsequently reverse-transcribed using a Firststrand cDNA Synthesis Kit (Thermo Scientific, USA) following the manufacturer's instructions. Primer sequences are listed in Additional file 11: Table S11. Realtime PCR was performed using a Roche LightCycler480 instrument (Roche, Basel, Switzerland) according to the manufacturer's protocol. The reaction system consisted of $5 \mu \mathrm{l}$ of SYBR Green (Toyobo), $0.3 \mu \mathrm{l}$ of each primer $(10 \mu \mathrm{M}), 1 \mu \mathrm{l}$ of diluted cDNA and $3.4 \mu \mathrm{l}$ of ultrapure water to a total volume of $10 \mu \mathrm{l}$. PCR included an initial denaturation step at $95^{\circ} \mathrm{C}$ for $30 \mathrm{~s}$, followed by 40 cycles at $95^{\circ} \mathrm{C}$ for $5 \mathrm{~s}, 57^{\circ} \mathrm{C}$ for $10 \mathrm{~s}$ and $72^{\circ} \mathrm{C}$ for $15 \mathrm{~s}$. Each sample was tested in triplicate. The $18 \mathrm{~S}$ ribosomal RNA gene was used as an internal control, and the comparative $2^{-\Delta \Delta C T}$ method was used to analyze expression levels of candidate genes.

\section{Supplementary information}

Supplementary information accompanies this paper at https://doi.org/10. 1186/s12864-020-06894-3.

Additional file 1: Table S1a, b. Gene structure optimize.

Additional file 2: Table S2a, b. Transcripts and its corresponding genomic mapping data.

Additional file 3: Table S3. SSR analysis result statistics.

Additional file 4: Table S4. Alternative polyadenylation analysis result. Additional file 5: Table S5a, b. Five kinds of alternative splicing events were identified.

Additional file 6: Table S6. The annotation of novel genes in database. Additional file 7: Table S7a, b. LncRNAs were identified.

Additional file 8: Table S8. The predicting target genes of IncRNAs based on location and co-expression relationship.

Additional file 9: Table S9. Differential alternative splicing analysis between smallest (S) and largest (L) subgroup.

Additional file 10: Table S10. The differentially expression transcript analysis data.

Additional file 11: Table S11. The nucleotide sequences of the primers for PCR.

\section{Abbreviations}

RNA: Ribonucleic acid; PCR: Polymerase chain reaction; SE: Skipped exon; MXE: Mutually exclusive exon; A5SS: Alternative 5' splice site:

A3SS: Alternative 3' splice site; RI: Retained intron

\section{Acknowledgements}

We thank International Science Editing (http://www.internationalscienceediting. com) for editing this manuscript.

\section{Authors' contributions}

$\mathrm{MH}$ designed this study, contributed to the original concept of the project and modified the paper. $\mathrm{HZ}$ performed the experiments. $\mathrm{HZ}$ wrote the paper. $H Z, H X, H L, X P, M X$ and $G Z$ performed the transcriptome analysis. All authors have read and approved the manuscript.

Funding

This study was supported by the Earmarked Fund for Modern Agro-industry Technology Research System (grant no. CARS-49) and the Science and Technology Planning Project of Guangdong Province, China (grant no. 2017B0303014052). The funders had no role in study design, data collection and analysis, decision to publish, or preparation of the manuscript.

\section{Availability of data and materials}

The raw sequence data reported in this paper have been deposited in the Genome Sequence Archive in National Genomics Data Center, Beijing Institute of Genomics, Chinese Academy of Sciences, under accession number CRA002619 that are publicly accessible at http://bigd.big.ac.cn/gsa. Data supporting the manuscript are also available in the supplementary information. The reference genome are used via the GigaDB database [59]. 


\section{Ethics approval and consent to participate}

All experiments were performed in agreement with the Experimental Animal Ethics of South China Sea Institute of Oceanology, Chinese Academy of Sciences.

\section{Consent for publication}

Not applicable.

\section{Competing interests}

The authors declare that they have no competing interests.

\section{Author details}

${ }^{1}$ CAS Key Laboratory of Tropical Marine Bio-resources and Ecology, Guangdong Provincial Key Laboratory of Applied Marine Biology, South China Sea Institute of Oceanology, Chinese Academy of Sciences, Guangzhou 510301, China. ${ }^{2}$ University of Chinese Academy of Sciences, Beijing 100049, China.

Received: 13 November 2019 Accepted: 7 July 2020

Published online: 13 July 2020

\section{References}

1. Zhang H, Zhao M, Yi XJ, Ou ZK, Li YG, Shi Y, et al. Characterization of the distal-less homologue gene, PfDlx, involved in regulating the expression of Pif in the pearl oyster, Pinctada fucata. Comp Biochem Physiol B-Biochem Mol Biol. 2017;212:51-8.

2. Sato Y, Komaru A. Pearl formation in the Japanese pearl oyster (Pinctada fucata) by $\mathrm{CaCO} 3$ polymorphs: pearl quality-specific biomineralization processes and their similarity to shell regeneration. Aquac Res. 2019;50(6): $1710-7$

3. Wang QH, Yang CY, Du XD, Liu XW, Sun RJ, Deng YW. Growth performance and biochemical composition of juvenile pearl oyster Pinctada martensii fed on artificial diets. Aquac Int. 2015;24(4):995-1005.

4. Liu WG, Yu ZH, Huang XD, Shi Y, Lin JS, Zhang H, et al. Effect of ocean acidification on growth, calcification, and gene expression in the pearl oyster, Pinctada fucata. Mar Environ Res. 2017:130:174-80.

5. Meng ZH, Zhang B, Liu BS, Li HM, Fan SG, Yu DH. High carotenoids content can enhance resistance of selected Pinctada fucata families to high temperature stress. Fish Shellfish Immunol. 2017;61:211-8.

6. Zhang $H$, Huang XD, Shi Y, Liu WG, He MX. Identification and analysis of an MKK4 homologue in response to the nucleus grafting operation and antigens in the pearl oyster, Pinctada fucata. Fish Shellfish Immunol. 2018; 73:279-87.

7. Munari M, Matozzo V, Chemello G, Riedl V, Pastore P, Badocco D, et al. Seawater acidification and emerging contaminants: a dangerous marriage for haemocytes of marine bivalves. Environ Res. 2019;175:11-21.

8. Jiao WQ, Fu XT, Dou JZ, Li HD, Su HL, Mao JX, et al. High-resolution linkage and quantitative trait locus mapping aided by genome survey sequencing: building up an integrative genomic framework for a bivalve mollusc. DNA Res. 2014;21(1):85-101.

9. Hao RJ, Du XD, Yang CY, Deng YW, Zheng Z, Wang QH. Integrated application of transcriptomics and metabolomics provides insights into unsynchronized growth in pearl oyster Pinctada fucata martensii. Sci Total Environ. 2019;666:46-56.

10. Stark R, Grzelak M, Hadfield J. RNA sequencing: the teenage years. Nat Rev Genet. 2019;20(11):631

11. Guan YY, He MX, Wu HB. Differential mantle transcriptomics and characterization of growth-related genes in the diploid and triploid pearl oyster Pinctada fucata. Mar Genomics. 2017;33:31-8.

12. Huang $S Q$, Ichikawa $Y$, Igarashi $Y$, Yoshitake $K$, Kinoshita S, Omori F, et al. Piwi-interacting RNA (piRNA) expression patterns in pearl oyster (Pinctada fucata) somatic tissues. Sci Rep. 2019;9(1):247.

13. Endo K, Takeuchi T. Annotation of the pearl oyster genome. Zool Sci. 2013; 30(10):779-80

14. Du XD, Fan GY, Jiao Y, Zhang H, Guo XM, Huang RL, et al. The pearl oyster Pinctada fucata martensii genome and multi-omic analyses provide insights into biomineralization. GigaScience. 2017;8:6

15. Abdel-Ghany SE, Hamilton M, Jacobi JL, Ngam P, Devitt N, Schilkey F, et al. A survey of the sorghum transcriptome using single-molecule long reads. Nat Commun. 2016;7:11706.
16. Zhang GQ, Sun M, Wang JF, Lei M, Li CJ, Zhao DJ, et al. PacBio full-length CDNA sequencing integrated with RNA-seq reads drastically improves the discovery of splicing transcripts in rice. Plant J. 2019;97(2):296-305.

17. Zuo CM, Blow M, Sreedasyam A, Kuo RC, Ramamoorthy GK, Torres-Jerez I, et al. Revealing the transcriptomic complexity of switchgrass by PacBio long-read sequencing. Biotechnol Biofuels. 2018;11:170

18. Zhu CH, Li XF, Zheng JY. Transcriptome profiling using Illumina- and SMRTbased RNA-seq of hot pepper for in-depth understanding of genes involved in CMV infection. Gene. 2018;666:123-33.

19. Jia D, Wang YX, Liu YH, Hu J, Guo YQ, Gao LL, et al. SMRT sequencing of full-length transcriptome of flea beetle Agasicles hygrophila (Selman and Vogt). Sci Rep. 2018;8(1):2197.

20. Wang B, Tseng E, Regulski M, Clark TA, Hon T, Jiao Y, et al. Unveiling the complexity of the maize transcriptome by single-molecule long-read sequencing. Nat Commun. 2016;7:11708.

21. Wang XM, Chen SY, Shi X, Liu DN, Zhao P, Yunze L, et al. Hybrid sequencing reveals insight into heat sensing and signaling of bread wheat. Plant J. 2019;98(6):1015-32.

22. Conesa A, Madrigal P, Tarazona S, Gomez-Cabrero D, Cervera A, McPherson A, et al. A survey of best practices for RNA-seq data analysis. Genome Biol. 2016;17:13.

23. De-Santis C, Jerry DR. Candidate growth genes in finfish-where should we be looking? Aquaculture. 2007;272(1-4):22-38.

24. Wang ZM, Liang FL, Huang RL, Deng YW, Li JH. Identification of the differentially expressed genes of Pinctada maxima individuals with different sizes through transcriptome analysis. Reg Stud Mar Sci. 2019;26:100512.

25. Liu HR, Zhang H, Pan XL, Xu M, Huang J, He MX. A high density genetic map by whole-genome resequencing for QTL fine-mapping and dissecting candidate genes for growth or sex traits in the pearl oyster (Pinctada fucata martensii). Aquaculture. 2020;519:734839.

26. Shi Y, He MX. Differential gene expression identified by RNA-Seq and gPCR in two sizes of pearl oyster (Pinctada fucata). Gene. 2014;538(2):313-22.

27. Sharon D, Tilgner H, Grubert F, Snyder M. A single-molecule long-read survey of the human transcriptome. Nat Biotechnol. 2013;31(11):1009-14.

28. Hackl T, Hedrich R, Schultz J, Forster F. Proovread: large-scale high-accuracy PacBio correction through iterative short read consensus. Bioinformatics. 2014;30(21):3004-11.

29. Li Y, Fang CC, Fu YH, Hu A, Li CC, Zou C, et al. A survey of transcriptome complexity in Sus scrofa using single-molecule long-read sequencing. DNA Res. 2018;25(4):421-37.

30. Elkon R, Ugalde AP, Agami R. Alternative cleavage and polyadenylation: extent, regulation and function. Nat Rev Genet. 2013;14(7):496-506.

31. Reddy AS, Marquez Y, Kalyna M, Barta A. Complexity of the alternative splicing landscape in plants. Plant Cell. 2013;25(10):3657-83.

32. Marquez Y, Brown JW, Simpson C, Barta A, Kalyna M. Transcriptome survey reveals increased complexity of the alternative splicing landscape in Arabidopsis. Genome Res. 2012;22(6):1184-95.

33. Bentley DL. Coupling mRNA processing with transcription in time and space. Nat Rev Genet. 2014;15(3):163-75.

34. Tilgner $\mathrm{H}$, Jahanbani F, Blauwkamp T, Moshrefi A, Jaeger E, Chen F, et al. Comprehensive transcriptome analysis using synthetic long-read sequencing reveals molecular co-association of distant splicing events. Nat Biotechnol. 2015;33(7):736-42.

35. Ren PR, Meng YX, Li BC, Ma XL, Si EJ, Lai Y, et al. Molecular mechanisms of acclimatization to phosphorus starvation and recovery underlying fulllength transcriptome profiling in barley (Hordeum vulgare L.). Front Plant Sci. 2018;9:500.

36. Rhoads A, Au KF. PacBio sequencing and its applications. Genom Proteom Bioinf. 2015:13(5):278-89.

37. Feng X, Jia YT, Zhu R, Chen K, Chen YF. Characterization and analysis of the transcriptome in Gymnocypris selincuoensis on the Qinghai-Tibetan plateau using single-molecule long-read sequencing and RNA-seq. DNA Res. 2019; 26(4):353-63.

38. Au KF, Sebastiano V, Afshar PT, Durruthy JD, Lee L, Williams BA, et al. Characterization of the human ESC transcriptome by hybrid sequencing. Proc Natl Acad Sci U S A. 2013;110(50):E4821-30.

39. Li QS, Li Y, Song JY, Xu HB, Xu J, Zhu YJ, et al. High-accuracy de novo assembly and SNP detection of chloroplast genomes using a SMRT circular consensus sequencing strategy. New Phytol. 2014;204(4):1041-9.

40. Xu ZC, Peters RJ, Weirather J, Luo HM, Liao BS, Zhang X, et al. Full-length transcriptome sequences and splice variants obtained by a combination of 
sequencing platforms applied to different root tissues of Salvia miltiorrhiza and tanshinone biosynthesis. Plant J. 2015;82(6):951-61.

41. Zhang Y, Nyong'A TM, Shi T, Yang PF. The complexity of alternative splicing and landscape of tissue-specific expression in lotus (Nelumbo nucifera) unveiled by Illumina- and single-molecule real-time-based RNA-sequencing. DNA Res. 2019;26(4):301-11.

42. Kirat D, Kato S. Monocarboxylate transporter 1 (MCT1) mediates transport of short-chain fatty acids in bovine caecum. Exp Physiol. 2006;91(5):835-44.

43. Distl O. Monocarboxylate transporters and their rôle in glucose homeostasis in ruminants. Vet J. 2007;173(1):16-7.

44. Thwaites DT, Anderson CM. H+-coupled nutrient, micronutrient and drug transporters in the mammalian small intestine. Exp Physiol. 2007;92(4):603-19.

45. Gumuslu E, Cine N, Ertan Gokbayrak M, Mutlu O, Komsuoglu Celikyurt I, Ulak G. Exenatide alters gene expression of neural cell adhesion molecule (NCAM), intercellular cell adhesion molecule (ICAM), and vascular cell adhesion molecule (VCAM) in the hippocampus of type 2 diabetic model mice. Med Sci Monit. 2016;22:2664-9.

46. Rathjen T, Yan X, Kononenko NL, Ku MC, Song K, Ferrarese L, et al. Regulation of body weight and energy homeostasis by neuronal cell adhesion molecule 1. Nat Neurosci. 2017;20(8):1096-103.

47. Horvath BM, Magyar Z, Zhang YX, Hamburger AW, Bakó L, Visser RG, et al. EBP1 regulates organ size through cell growth and proliferation in plants. EMBO J. 2006;25:4909-20.

48. Kowalinski E, Bange G, Wild K, Sinning I. Expression, purification, crystallization and preliminary crystallographic analysis of the proliferationassociated protein Ebp1. Acta Crystallogr Sect F Struct Biol Cryst Commun. 2007;63(Pt 9):768-70.

49. Kittler R, Putz G, Pelletier L, Poser I, Heninger AK, Drechsel D, et al. An endoribonuclease-prepared siRNA screen in human cells identifies genes essential for cell division. Nature. 2004;432(7020):1036-40.

50. Amiri M, Yousefnia S, Seyed Forootan F, Peymani M, Ghaedi K, Nasr Esfahan $\mathrm{MH}$. Diverse roles of fatty acid binding proteins (FABPs) in development and pathogenesis of cancers. Gene. 2018:676:171-83.

51. Hao RJ, Wang ZM, Yang CY, Deng YW, Zheng Z, Wang QH, et al. Metabolomic responses of juvenile pearl oyster Pinctada maxima to different growth performances. Aquaculture. 2018;491:258-65.

52. Spurlock ME. Regulation of metabolism and growth during immune challenge: an overview of cytokine function. J Anim Sci. 1997;75(7):1773-83.

53. Wu TD, Watanabe CK. GMAP: a genomic mapping and alignment program for mRNA and EST sequences. Bioinformatics. 2005;21 (9):1859-75.

54. Simao FA, Waterhouse RM, loannidis P, Kriventseva EV, Zdobnov EM BUSCO: assessing genome assembly and annotation completeness with single-copy orthologs. Bioinformatics. 2015;31(19):3210-2.

55. Zhang HM, Liu T, Liu CJ, Song SY, Zhang XT, Liu W, et al. AnimalTFDB 2.0: a resource for expression, prediction and functional study of animal transcription factors. Nucleic Acids Res. 2015:43(D1):D76-81.

56. Foissac S, Sammeth M. ASTALAVISTA: dynamic and flexible analysis of alternative splicing events in custom gene datasets. Nucleic Acids Res. 2007; 35:W297-9.

57. Xie C, Mao XZ, Huang JJ, Ding Y, Wu JM, Dong S, et al. KOBAS 2.0: a web server for annotation and identification of enriched pathways and diseases. Nucleic Acids Res. 2011;39:W316-22.

58. Anders S, Huber W. Differential expression analysis for sequence count data. Genome Biol. 2010;11(10):R106.

59. Du XD, Fan GY, Jiao Y, Zhang H, Guo XM, Huang RL, et al. Supporting data for "the pearl oyster Pinctada fucata martensii genome and multi-omic analyses provide insights into biomineralization". GigaScience Database. 2017;6(8):gix059. https://doi.org/10.5524/100240.

\section{Publisher's Note}

Springer Nature remains neutral with regard to jurisdictional claims in published maps and institutional affiliations.

Ready to submit your research? Choose BMC and benefit from:

- fast, convenient online submission

- thorough peer review by experienced researchers in your field

- rapid publication on acceptance

- support for research data, including large and complex data types

- gold Open Access which fosters wider collaboration and increased citations

- maximum visibility for your research: over $100 \mathrm{M}$ website views per year

At $\mathrm{BMC}$, research is always in progress.

Learn more biomedcentral.com/submissions 